\title{
The politics of progressive income taxation with incentive effects
}

\author{
Philippe De Donder* \\ University of Toulouse (IDEI and GREMAQ) \\ and \\ Jean Hindriks** \\ CORE and Queen Mary, University of London
}

First version July 2000 - This version January 2002

\begin{abstract}
This paper studies majority voting over quadratic taxation and investigates under which conditions marginal progressivity emerges as a voting outcome. In our model with endogenous income, there is no majority (Condorcet) winning tax schedule. We then investigate less demanding political equilibrium concepts in order to see under which conditions the set of equilibria is composed only of progressive tax functions. We follow three strategies: (i) reduction of the policy space to the tax functions that are ideal for some voter; (ii) elimination of weakly dominated strategies and use of mixed strategies in a standard Downsian two-party competition game; (iii) assumption that political parties interact repeatedly and care about the size of their majority. Although each approach captures a different aspect of political behavior, all point to the same (simulation-based) conclusion that progressivity is more likely to emerge for most distributions of abilities and that it is actually the only possible voting outcome if the distribution is sufficiently concentrated around the middle.
\end{abstract}

JEL classification: D72

Keywords: Majority Voting; Income taxation; Tax Progressivity.

*Manufacture des Tabacs, 21 allée de Brienne, 31000 Toulouse, France, Email: dedonder@cict.fr

** Queen Mary, University of London, Mile End Road, London E1 4NS, England. Tel. (44)-020-78827807, Email:j.hindriks@qmw.ac.uk. 


\section{Introduction}

Under which conditions do marginally progressive income tax schedules emerge? The economic literature has tried to answer this question by resorting either to a normative or a positive approach. The normative approach assumes that tax policies are chosen by a benevolent planner, whose objective is to maximize social welfare under informational and incentive constraints. Unfortunately, this approach has proven inconclusive on the shape of the optimal tax function (for a recent account, see Myles, 2000). A notable exception is when the planner's objective is to choose a "fair" tax schedule. Indeed, Young (1990) has shown that the equal sacrifice requirement implies progressive taxation.

The positive approach, to whom this paper clearly belongs, stresses the fact that tax schedules are, directly or indirectly, chosen democratically by self-interested voters. The primary objective of the paper is to provide conditions for progressivity (in the sense of convex tax functions) to emerge as a voting outcome. More precisely, we analyze voting outcomes and their dependence on the underlying distribution of abilities in presence of tax disincentives. We specify the disincentive effect of taxation in such a way that the revenue-maximizing tax schedule is regressive. The idea is that if progressivity can emerge as politicial equilibrium in this most unfavorable environment, then modifying tax disincentives can only make progressivity more likely.

The main difficulty in applying voting theory to (non-linear) income taxation resides in the multi-dimensionality of the policy space and in its consequences on the existence of an equilibrium. It is well known that the aggregation, by means of majority voting, of transitive individual preferences on a multi-dimensional set of options often results in a non-transitive social preference. In most cases, there is no majority (or Condorcet) winner, i.e. no option that is preferred by a majority to any other feasible option. 
Like many papers in the domain ${ }^{1}$, we address this issue by reducing the dimensionality of the policy space. More precisely, we confine the analysis to quadratic tax schedules, including either convex, concave or linear tax functions (like Roemer,1999 and Cukierman and Meltzer, 1991). This is of course an important restriction since it rules out tax schedules with nonmonotone marginal tax rates (like in Roell, 1997). However it allows us to reduce the dimensionality of the voting space to a manageable number. This restriction is not sufficient in itself to guarantee the existence of a majority winner. Indeed Cukierman and Meltzer (1991) using a similar model show that rather strong conditions are required for the existence of a majority winner and for that equilibrium to involve progressive taxes. So in spite of the restriction to quadratic tax schedules and unless we impose very strong additional restrictions, there is no majority equilibrium and vote cycles are almost inevitable.

We use three different approaches to bypass the problem of the non existence of a majority winner. ${ }^{2}$ In the first approach, we restrict the policy space to tax schedules that are ideal for some voter (Section 3). We show by means of simulations that there exists a majority equilibrium on this restricted voting space and that it involves progressivity when the distribution of abilities is concentrated around the middle. ${ }^{3}$ In the second approach, developed in Section 4, we keep the entire policy space but adopt less demanding political equilibrium concepts in a simultaneous two-party competition game, namely the uncovered set (elimination of weakly domi-

\footnotetext{
${ }^{1}$ Romer (1975) reduces the policy space to linear tax schedules and obtains a Condorcet winner involving average progressivity (see also Roberts (1977)). Berliant and Gouveia (1994) introduce uncertainty over the income distribution and then use the ex-ante budget balance requirement to reduce the policy space so that a Condorcet winner exists. Snyder and Kramer(1988) assume that candidates cannot credibly commit to implement something different from their most-preferred policy and thus restrict the policy space to the policies that are ideal for some voter.

${ }^{2}$ We do not investigate in this paper the probabilistic voting approach according to which voters who are the most affected by a tax change are the more likely to vote. This sort of randomness facilitates the existence of a majority equilibrium (see Hinich et al, 1972).

${ }^{3}$ Snyder and Kramer (1988) obtain a similar result with a different model.
} 
nated strategies) and the bipartisan set (mixed-strategy equilibrium). ${ }^{4}$ In the third approach we consider a sequential two-party competition due to Kramer (1977) in which political parties propose policies that maximize their vote shares. ${ }^{5}$ We determine the vote-maximizing trajectory and show that it converges to a relatively small cycle.

Simulating the model for different distributions of abilities, we show that the second and third approaches narrow down significantly the set of equilibria and tend to select mostly progressive taxes when the distribution of abilities is concentrated around the middle.

\section{The Model}

\subsection{Tax Functions}

Following the optimal income tax tradition, we consider a two-good economy (consumption and labor) populated by a large number of individuals who differ only in their ability. Each individual is characterized by her ability to earn income, $w \in[0,1]$. The distribution of ability in the population is described by a strictly increasing cumulative distribution function $F$ on $[0,1]$, so that $F(w)$ is the fraction of the population with ability less than or equal to $w$. The average ability level is

$$
\bar{w}=\int_{0}^{1} w d F(w)
$$

and the median ability level is

$$
w_{m}=F^{-1}\left(\frac{1}{2}\right) \text {. }
$$

We assume throughout that $w_{m} \leq \bar{w}$ in order to generate positively skewed income distributions as typically observed in real world. Individuals

\footnotetext{
${ }^{4}$ See De Donder et al.(2000) for a presentation and a set-theoretical comparison of these different solution concepts. See also De Donder(2000) for an application to redistributive issues. All these concepts are Condorcet-consistent in the sense that they uniquely select the Condorcet winner whenever it exists.

${ }^{5}$ Hettich and Winer (1988) follow a similar approach in a model in which political parties propose nonlinear taxes to maximize their vote share. However work disincentives are not present in their model.
} 
choose the amount of labor they sell on a competitive market and receive a wage rate equal to their ability. The production sector exhibits constantreturns-to-scale so that the wage rate is constant. Then an individual with ability $w \neq 0$ who supplies $y / w$ units of labor earns pre-tax income $y$. Her after-tax income is

$$
x(y, t)=y-t(y)
$$

where $t(y)$ is a continuous tax function $t: R_{+} \rightarrow R$. Note that we allow for negative taxes.

Definition 1. A tax function is feasible if it satisfies the following conditions,

$$
\begin{gathered}
t(y) \leq y \quad \text { for all } \quad y \in R_{+} \\
0 \leq t^{\prime}(y) \leq 1 \quad \text { for all } \quad y \in R_{+} \\
\int_{0}^{1} t(y(w)) d F(w)=0
\end{gathered}
$$

Condition (4) says that tax liabilities cannot exceed taxable income. Condition (5) implies that both tax liabilities and after-tax income are nondecreasing functions of pre-tax income. ${ }^{6}$ The budget balance condition (6) means that income taxation is purely redistributive (i.e., zero revenue requirement).

Our primary objective is to understand when progressive taxation emerges as a voting outcome. We adopt the following definition of progressivity. ${ }^{7}$

Definition 2: A tax schedule is (marginally) progressive if and only if $t(y)$ is a convex function (i.e. marginal tax rates are monotonically increasing).

\footnotetext{
${ }^{6}$ This condition is usually derived instead of assumed in the optimal income tax literature with endogenous labor supply.

${ }^{7}$ As suggested by one referee by allowing for negative taxes our definition of progressivity (marginal progressivity) does not necessarily correspond to the more usual definition of progressivity in terms of increasing average tax rates (average progressivity) which gives a better indication of the level of redistribution. But since the objective of this paper is to understand the prevalence of (weakly) convex tax function rather than the level of redistribution, the concept of marginal progressivity seems more appropriate.
} 
The set of potential tax schedules is infinitely dimensional. To limit the voting problem to a manageable number of dimensions we shall thereafter restrict attention to the quadratic income tax function: ${ }^{8}$

$$
t(y)=-c+b y+a y^{2}
$$

where $c \geq 0$ is the uniform lump-sum transfer, $b$ is the flat tax parameter (with $0 \leq b \leq 1$ ) and $a$ is the progressivity tax parameter, with $a>0$ indicating a (marginally) progressive income tax and $a<0$ representing a (marginally) regressive one. As will become clear shortly, the feasibility conditions (4) and (5) impose the following lower and upper bounds on the progressivity parameter: $\frac{-b}{2} \leq a \leq \frac{1-b}{2}$. Essentially, the upper bound on progressivity ensures that the marginal tax rate is less than one at the top (and thus everywhere) and the lower bound on regressivity guarantees that marginal tax rate is positive at the top (and thus everywhere). Combining (6) and (7) yields

$$
c=b \bar{y}+a \bar{y}_{2}
$$

where $\bar{y}=\int y(w) d F(w)$ and $\bar{y}_{2}=\int y^{2}(w) d F(w)$. Hence, tax policies are bidimensional. Let the set of feasible tax policies be $T=\left\{(a, b) \in\left[\frac{-b}{2}, \frac{1-b}{2}\right] \times[0,1]\right\}$. Plugging (7) and (8) into (3) the after-tax income (consumption) of an individual with pre-tax income $y$ resulting from a tax policy $(a, b) \in T$ is given by

$$
x=\bar{y}+(1-b)(y-\bar{y})-a\left(y^{2}-\bar{y}_{2}\right)
$$

\section{$2.2 \quad$ Preferences}

The underlying preferences of any individual with ability $w$ are represented by a utility function $u(x, y ; w)$. Given the tax policy $(a, b)$, an individual with ability $w$ chooses pre-tax income $y$ that maximizes $u(x, y ; w)$ subject to (9). Throughout we assume the following quasi-linear utility function,

\footnotetext{
${ }^{8}$ The quadratic tax function has been studied before in a voting context by Cukierman and Meltzer (1991) with endogenous incomes and by Roemer (1999) with exogenous incomes. Note that quadratic taxation can be viewed as a second-order Taylor expansion of a more general class of schedules.
} 


$$
u(x, y ; w)=x-\frac{1}{2}\left(\frac{y}{w}\right)^{2} .
$$

This formulation of preferences simplifies the analysis as there is no income effect on the labor supply decision (all income effects are absorbed by consumption) and everyone chooses to work (because the marginal disutility of labor is zero when not working). While this is a highly restrictive specification of preferences, it captures in a simple manner the incentive effects of taxes (consumption-leisure trade-off), thus making it more general than models with fixed income (e.g., Roemer, 1999). It is also considerably more tractable than the general specification of Cukierman-Meltzer (1991), allowing us to obtain clear, intuitive results. ${ }^{9}$

Using this specification of preferences, for each tax policy $(a, b) \in T$ an individual with ability $w$ will choose a pre-tax income ${ }^{10}$

$$
y(a, b ; w)=(1-b) \frac{w^{2}}{1+2 a w^{2}} .
$$

Hence marginal tax progressivity $(a>0)$ reduces the pre-tax income of everyone. Moreover because progressivity discourages more the labor supply of high-ability than low-ability individuals, the dispersion of pretax income decreases. Note also that for each $(a, b) \in T$ pre-tax income is increasing with ability. Therefore for any admissible tax schedule the ranking of incomes is the same as the ranking of abilities.

Given the optimal labor choices the upper bound on progressivity and the lower bound on regressivity ensure that any tax policy $(a, b) \in T$ satisfies the conditions $(4)$ to $(6) .{ }^{11}$

\footnotetext{
${ }^{9}$ Some recent empirical studies also support our quasi-linearity assumption. For instance Blundell et al. (1998) report very low income elasticities of labour supply.

${ }^{10} \mathrm{With}$ regressive taxation the budget set is not convex but the lower bound imposed on the degree of regressivity is sufficient relative to the degree of concavity of the utility function to ensure that the optimal labor supply choice is unique for all ability levels $w \in[0,1]$ and tax policies $(a, b) \in T$.

${ }^{11}$ The proof of this statement is available upon request.
} 
Resorting to numerical simulations for various ability distributions, we further get that in this model the lump sum tranfer $c$ is globally maximized with regressive taxes. So the poor would prefer marginal regressivity. This is a consequence of the labor-enhancing effect of regressive taxation. Lower tax rates on the rich induce them to earn more and eventually pay more taxes that can then be redistributed to the poor.

Preferences over tax policies are given by the indirect utility function. Substituting (3), (7) and (11) into (10) the indirect utility function of an individual with ability $w$ over a tax policy $(a, b)$ is

$$
v(a, b ; w)=c+(1-b)^{2} \frac{\omega(a)}{2} .
$$

where $\omega(a)=\frac{w^{2}}{1+2 a w^{2}}$ and from (8) $c$ is a function of the tax policy $(a, b) \in T$ and the distribution of ability $F(w)$. Since $\omega(a)$ is increasing in $w$ for all $(a, b) \in T$ it follows that higher ability individuals get higher utility for any admissible tax schedule.

\subsection{Voting over quadratic tax schemes}

We now turn to the voting problem over (non-linear) tax policies $(a, b) \in T$. A majority (or Condorcet) winning tax policy is a pair $(a, b)$ that is preferred by a majority of individuals to any other feasible pair $\left(a^{\prime}, b^{\prime}\right) \in T$. The natural first step is to examine if such a majority winning tax policy exists. Previous research has delivered several sufficient conditions for the existence of majority voting equilibrium. When the decision is unidimensional either single-crossing or single-peakedness of preferences is sufficient (Roberts 1977; Gans and Smart 1996). Unfortunately single-crossing and single-peakedness do not extend to our two-dimensional issue space since there is in general no way to rank multi-dimensional tax schedules in a transitive order.

The absence of voting equilibrium when taxation is multidimensional is more readily described when income is fixed. In this case, Marhuenda and 
Ortuno-Ortin(1995) have shown that, starting with any tax schedule, there always exists a majority support for more (marginal) progressivity when the median income is lower than the mean income. This move towards more progressivity is supported by the low income individuals, who thereby shift the burden of taxation towards higher income tax payers. On the other hand, Hindriks(2001) has also shown, using quadratic tax functions, that there always exists a majority support for less progressivity. This tax reform is supported by a coalition of the rich and the poor as a way to shift the burden of taxation towards the middle income group. Put together, these two results imply inevitable voting cycles between progressive and regressive taxes $^{12}$ along which majority coalitions of the poor and the middle class alternate with majority coalitions of the poor and the rich. ${ }^{13}$

Allowing for endogenous income, and thus distortionary taxation, does not alleviate the existence problem of a Condorcet winner. ${ }^{14}$ Facing this problem, one possibility is to find conditions over ability distributions and voter preferences such that a Condorcet winner emerges. This is the route taken by Cukierman and Meltzer (1991) with quadratic tax functions. The conditions they obtain seem unsurprisingly very restrictive. ${ }^{15}$

\footnotetext{
${ }^{12}$ Notice that it is not possible to follow Gans and Smart (1996) in restricting our analysis to a family of non-linear tax schedules that cross only once so as to reduce the multi-dimensional tax schedules into a one-dimensional index of progressivity (see also Berliant and Gouveia, 1994). Indeed, doing so would create a bias in favor of progressivity and the middle-class since a coalition of the rich and poor can only form in our model by proposing a tax schedule crossing twice from below the existing one.

${ }^{13}$ Both Marhuenda and Ortuno-Ortin(1995) and Hindriks(2001) assume that it is always possible to construct either more progressive or less progressive tax scheme than the existing one. Making some reasonable restrictions on the admissible tax schedule, De Donder and Hindriks(2002) show that with fixed income individuals display corner preferences over tax schedules and that it is then easier to obtain a Condorcet winner since many deviations are ruled out. They also give necessary and sufficient conditions for a Condorcet winner to exist, and show that if it exists it entails maximum progressivity.

${ }^{14}$ Indeed, as we show in section 3 , with distortionary taxation individuals have interior preference for tax schedules. This makes the existence of a Condorcet winner more difficult to obtain than with fixed income.

${ }^{15}$ They do not provide examples of preferences and distribution functions satisfying these conditions. Indeed, the non-existence of a Condorcet winner in our highly stylized model (which uses the same quadratic tax schedules and very simple preferences and ability distributions) casts doubt on the possibility for their conditions to be met in a more general context.
} 
This paper investigates other ways to face the existence problem of a Condorcet winner. More precisely, the following three sections offer three different approaches. In section 3, we reduce the set of admissible tax functions to those which are most preferred by some voter. In section 4, we introduce simultaneous political competition between two parties only interested in getting elected, and we study both their weakly undominated strategies (uncover set) and their mixed strategy equilibrium (bipartisan set). In section 5 , we allow for sequential political competition and study the trajectory of equilibrium strategies. For each approach we analyze the equilibrium outcomes and their comparative statics with respect to changes in the underlying distribution of abilities. The central question is under which conditions marginal progressivity is more likely to emerge as a voting outcome.

Given the complexitity of the analysis we can only provide simulation results. Throughout we shall adopt the beta distribution as the distribution of ability. The beta distribution has two parameters $(\alpha>0$ and $\beta>0)$, varying which can generate a wide variety of density functions with support $[0,1] .{ }^{16}$ The beta distribution has mean $\alpha /(\alpha+\beta)$ and variance $\alpha \beta /[(1+$ $\left.\alpha+\beta)(\alpha+\beta)^{2}\right]$. If $\alpha>1$ and $\beta>1$ the distribution is unimodal. If $\alpha<1$ and $\beta<1$ it is $\mathrm{U}$-shaped, while if $\alpha=\beta=1$ it is uniform. The degree of skewness increases with the difference $|\alpha-\beta|$. The beta function is symmetric if $\alpha=\beta$, positively skewed if $\alpha>\beta$ and negatively skewed if $\alpha<\beta$. If $\alpha \leq 1$ and $\beta>1$ the density is J-shaped (monotonically increasing) whereas if $a>1$ and $b \leq 1$ it is the opposite (monotonically decreasing). Increasing both $\alpha$ and $\beta$ increases the density around the median.

\footnotetext{
${ }^{16}$ The beta distribution has density $f(w)=\frac{1}{B(\alpha, \beta)} w^{\alpha-1}(1-w)^{\beta-1}(0 \leq w \leq 1)$ where $B(\alpha, \beta)$ is the beta function that is defined by $B(\alpha, \beta)=\int_{0}^{1} x^{\alpha-1}(1-x)^{\beta-1} d x$ for $\alpha>0$ and $\beta>0$.
} 


\section{Reduction of the policy space}

In this section, we restrict the policy space to the set of feasible tax schedules that are most-preferred by some voter. This assumption seems a natural way to reduce the policy space, and has been adopted by Snyder and Kramer (1988) enabling them to obtain a Condorcet winner with progressive taxation. ${ }^{17}$ We can also defend this restriction along the line of the CitizensCandidates models by assuming that candidates, emerging from the set of voters, cannot credibly commit to choose a policy different from their most preferred policy (Osborne and Slivinsky 1996, Besley and Coate 1997).

As an illustration, in Figure 1 we have represented the most preferred tax functions of the electorate for the beta distribution of ability with parameters $\alpha=2, \beta=5$ (i.e., unimodal and skewed to the right). ${ }^{18}$ The lowest-ability individual prefers the policy that maximizes $c$ (i.e., the peak of the Laffer surface). This is the most regressive tax schedule, with the lowest $a<0$ and the highest $b$, namely $\left(a_{1}, b_{1}\right)=(-0.28,0.55)$. The second lowest-ability individual prefers the point just below $\left(a_{2}, b_{2}\right)=(-0.25,0.50)$ and so on moving downwards as ability increases, trading off progressively lower $b$ for higher $a$. Then we reach the median ability individual who prefers $\left(a_{m}, b_{m}\right)=(0.425,0.15)$. Proceeding further we reach a voter close to the mean who prefers a policy with $b=0$ and maximum progressivity $a=1 / 2$. From that point on we move to the left along the horizontal axis as ability increases, involving less and less progressivity as a goes down to zero. ${ }^{19}$ Ordering all the bliss points from left to right by increasing ability we obtain that each voter displays single-peaked preferences over the resulting set of bliss points. Therefore it follows from the median voter theorem that

\footnotetext{
${ }^{17}$ Their model differs however from ours in the sense that individuals do not respond to taxation by substituting untaxable leisure to taxable labor, but rather by working in an untaxed sector with lower wage rate.

${ }^{18}$ For this example, $a$ and $b$ vary by increment of 0.005 respectively in $\left[-\frac{b}{2}, \frac{1-b}{2}\right] \times[0,1]$.

${ }^{19}$ Calculations with other ability distributions reveal a similar pattern of blisspoints with a willingness to trade off less $b$ for more $a$ as ability rises until $b=0$, then to decrease $a$ as ability further increases until the no taxation point $(a, b)=(0,0)$ is reached.
} 
the point preferred by the median ability individual is a majority winner. ${ }^{20}$ Since the median voter is in general in favor of progressivity we get a first possible explanation to the prevalence of progressive taxation. Existence of voting equilibrium over the set of blisspoints also obtains for different underlying distributions of abilities. However when the distribution of ability changes the median voter may change as well as the impact of tax on aggregate revenue, modifying the equilibrium outcome as illustrated in Table 1.

Table 1. Majority winner $\left(a_{m}, b_{m}\right)$ over bliss points for various $\operatorname{beta}(\alpha, \beta)$

\begin{tabular}{c|cc}
\hline $\operatorname{Beta}(\alpha, \beta)$ & $a_{m}$ & $b_{m}$ \\
\hline 1,1 & 0.275 & 0 \\
1,2 & -0.075 & 0.35 \\
\hline 2,2 & 0.25 & 0 \\
2,3 & 0.475 & 0 \\
2,4 & 0.5 & 0 \\
2,5 & 0.425 & 0.15 \\
\hline $2.5,2.5$ & 0.225 & 0 \\
$2.5,3$ & 0.35 & 0 \\
$2.5,4$ & 0.5 & 0 \\
$2.5,5$ & 0.5 & 0 \\
\hline 3,3 & 0.225 & 0 \\
3,4 & 0.325 & 0 \\
3,5 & 0.5 & 0
\end{tabular}

Table 1 reveals that in most cases the median voter prefers progressivity (although the degree of progressivity may vary with the parameters of the distribution of abilities). The only exception is the linearly decreasing

\footnotetext{
${ }^{20}$ In the course of our research, Robin Boadway and John Weymark have brought to our attention that a similar existence result has been derived by Roell (1997) under quasi-linear preferences and one additional condition which she called minimum utility restriction. This restriction requires that the lowest ability individual prefers the consumption-income bundle of the median voter to the zero bundle. It appears however that this condition cannot be satisfied in our model since the lowest ability is zero (so, making it infinitely harmful for this individual to reach the median income level). Roell's result is also driven by the fact that in her model higher ability individuals prefer tax schedules that induce more effort from everyone (see Theorem 6). This is not consistent with our model in which the poor tend to support marginal regressivity which elicits more effort than the marginal progressivity favored by the middle-class. Another key difference between the two models is the feasible set. For instance in Roell the equilibrium outcome involves negative marginal tax rates for some individuals below the median, which is not feasible in our model.
} 
distribution of abilities (i.e., $\operatorname{Beta}(1,2))$ for which the median ability is so low that the median voter prefers regressivity like the low-ability voters.

The existence of a majority winner over the bliss points deserves some explanation. It is essentially due to the fact that the rich and the poor having diametrically opposed ideal policies (pure redistribution) can no longer compromise on a policy that is not optimal for one of them to defeat the policy which is optimal for the median voter. This prevents the formation of a coalition of the extremes and gives the decisive power to the median voter. The remaining voters are evenly paired off against one another regarding any move from the policy that is most-preferred by the voter with median ability. ${ }^{21}$

In the following section, instead of restricting the vote to the most preferred tax functions we adopt weaker solution concepts in the context of a standard Downsian political competition game.

\section{Elimination of weakly dominated policies}

We consider a Downsian voting game with two political parties competing to win the election. Both parties simultaneously announce their tax schedule which they commit to implement if elected. Each individual then votes for the party whose platform is better according to her preferences. The party receiving the most votes wins the election and imposes its platform as the choice of the polity. In the event of ties, a fair coin decides which party wins the election. Note that in contrast with Section 3, it is assumed here that candidates can commit to any policy.

\footnotetext{
${ }^{21}$ To see this, fix the interior blisspoint of the median individual (with income $y_{m}>0$ ) and suppose a small change in $a$ from that point. Let $\frac{d b}{d a}$ be the associated change in $b$ required to maintain $c$ constant. Then from the first-order condition and the envelope theorem we have, $\partial v\left(y_{m}\right) / \partial a=-\left(\frac{d b}{d a}+y_{m}\right) y_{m}=0$ which implies $\frac{d b}{d a}=-y_{m}$. Using this fact, it then follows that $\partial v(y) / \partial a=-\left(y-y_{m}\right) y \gtrless 0$ for all $y \lessgtr y_{m}$. Hence, voters on each side of the median income disagree about the desired direction of change from the policy preferred by the median voter.
} 
The majority preference $P$ over any pair of tax schedules $\left(t_{1}, t_{2}\right) \in T^{2}$ is given by,

$$
\begin{array}{ccc}
t_{1} P t_{2}: & n\left(t_{1}, t_{2}\right)>n\left(t_{2}, t_{1}\right) \\
t_{2} P t_{1}: & n\left(t_{1}, t_{2}\right)<n\left(t_{2}, t_{1}\right),
\end{array}
$$

where $n\left(t_{1}, t_{2}\right)=\#\left\{w \in[0,1]: v\left(t_{1} ; w\right)>v\left(t_{2} ; w\right)\right\}$ is the number of voters who (weakly) prefer $t_{1}$ to $t_{2}$, and $n\left(t_{2}, t_{1}\right)=\#\left\{w \in[0,1]: v\left(t_{1} ; w\right)<\right.$ $\left.v\left(t_{2} ; w\right)\right\}$ is the number of voters who prefer $t_{2}$ to $t_{1} \cdot{ }^{22}$

Assuming an odd number of voters, the majority preference relation is a binary relation satisfying the asymmetry and completeness properties of a tournament.

The objectives assigned to the parties are crucial. We suppose that parties are only interested in winning the election and that they derive no intrinsic utility from the platform chosen (i.e., no ideology). Moreover parties are indifferent about the size of their majority: having a bare majority they attach no utility to any extra vote (we relax this assumption in the next section). Given that parties can choose among the same set of feasible policies, we can represent this electoral competition by a symmetric two-player zero-sum game $G=(T, T, U)$ where each party's utility is

$$
U\left(t_{1}, t_{2}\right)=\left\{\begin{array}{rlr}
1 & : & t_{1} P t_{2} \\
-1 & : & t_{2} P t_{1} \\
0 & : & t_{1}=t_{2}
\end{array}\right.
$$

This game, called the majority game, has a unique Nash equilibrium in pure strategies if and only if there exists a Condorcet winner. Formally, $t^{*} \in T$ is a Condorcet winner if and only if $t^{*} P t$ for all $t \in T \backslash\left\{t^{*}\right\}$. In this case, both parties choose the Condorcet winner as a strategy.

But we know that there is no Condorcet winner in the (unrestricted) two-dimensional policy space. It follows that the game does not have any equilibrium in pure strategies: each party could win the election if it knew which policy is chosen by the other party.

\footnotetext{
${ }^{22}$ We rule out indifference since it is a very unlikely event.
} 
However, the absence of a Condorcet winner does not imply that any policy is equally likely to be selected by any party. First, we do not expect any party to select a Pareto dominated policy.

It is also unlikely that any party will play weakly dominated policies. A policy $t^{\prime}$ is weakly dominated by policy $t^{\prime \prime}$ if $U\left(t^{\prime}, t\right) \leq U\left(t^{\prime \prime}, t\right)$ for all $t \in T$, with a strict inequality for at least one $t$. Reformulated in our voting context, $t^{\prime \prime}$ weakly dominating $t^{\prime}$ means that $t^{\prime \prime}$ beats $t^{\prime}$ as well as any policy $t$ that $t^{\prime}$ can beat.

In the social choice literature, this dominance relation is also called the covering relation (see Miller, 1980). Formally, given a tournament $(T, P)$, a policy $t^{\prime} \in T$ is covered whenever there exists some other policy $t^{\prime \prime} \in T$ such that $t^{\prime \prime} P t^{\prime}$ and $\left\{t \in T: t^{\prime} P t\right\} \subseteq\left\{t \in T: t^{\prime \prime} P t\right\}$. The set of options that are not covered (for the majority preference relation $P$ ) is called the uncovered set, denoted by $U C(T, P)$. Since the covering relation is equivalent to the weak dominance relation in our setting, the uncovered set is precisely the set of weakly undominated pure strategies of the two-party zero-sum game $G=\{T, T, U\}$. Given that any Pareto-dominated policy is covered, the uncovered set is a subset of the Pareto set. ${ }^{23}$

A nice feature of the uncovered set is that it is not empty even when a majority winner fails to exist. On the other hand, in absence of Condorcet winner any uncovered policy will be defeated by other policies, even possibly by policies not belonging to the uncovered set. Therefore the uncovered set does not describe the policies that should be chosen. Instead it means that parties can perform at least as well by restricting themselves to the uncovered policies.

We can further restrict the set of interesting strategies by looking at the Nash equilibrium in mixed strategies of this game. ${ }^{24}$ Laffond et al. (1993)

\footnotetext{
${ }^{23}$ If $t$ is Pareto-dominated by $t^{\prime}, t^{\prime}$ has a better position than $t$ in every individual preference ordering. Hence, using majority voting, $t^{\prime}$ beats all the options that $t$ beats, which means that $t^{\prime}$ covers $t$.

${ }^{24}$ See Laslier(2000) for an interpretation of electoral mixed strategies
} 
have shown that the finite and symmetric majority game $G=(T, T, U)$ has a unique equilibrium in mixed strategies. They call the support of this unique equilibrium the bipartisan set denoted $B P(T, P) .{ }^{25}$ It can be shown that the bipartisan set is a subset of the uncovered set and thus a more discriminating solution concept (see Banks et al., 1998). Furthermore, both the uncovered set and the bipartisan set reduce to the Condorcet winner whenever the latter exists.

Given these definitions, we can now compute the uncovered set and the bipartisan set as a function of the abilities' distribution. The results are reported in Table 2 for the beta distribution with various parameters $(\alpha, \beta)^{26}$. For each solution concept (including the Kramer cycle presented in Section 5 ), the first column gives the size of the solution set expressed in percentage of the feasible set and the second column gives the proportion of the solution set that is composed of (weakly) progressive tax schedules.

Table 2. Uncovered set (UC), Bipartisan set (BP) and Kramer cycle

\begin{tabular}{|c|c|c|c|c|c|c|}
\hline \multirow{2}{*}{$\operatorname{Beta}(\alpha, \beta)$} & \multicolumn{2}{|c|}{$\overline{\mathrm{UC}}$} & \multicolumn{2}{|c|}{$\mathrm{BP}$} & \multicolumn{2}{|c|}{ Kramer } \\
\hline & $\%$ & \%progr & $\%$ & \%prog & $\%$ & $\%$ prog \\
\hline 1,1 & 16.9 & 64.8 & 5 & 76.2 & 2.62 & 45.4 \\
\hline 1,2 & 0.7 & 0 & 0.7 & 0 & 0.7 & 0 \\
\hline 2,2 & 4.8 & 85 & 1.7 & 100 & 1.7 & 100 \\
\hline 2,3 & 15.7 & 80.3 & 6.4 & 96.3 & 2.86 & 91.7 \\
\hline 2,4 & 18.3 & 79.2 & 7.9 & 93.9 & 1.4 & 66.7 \\
\hline 2,5 & 9.3 & 71.8 & 4 & 88.2 & 1.7 & 28.6 \\
\hline $2.5,2.5$ & 3.3 & 100 & $\begin{array}{l}1.7 \\
\end{array}$ & 100 & 0.9 & 100 \\
\hline $2.5,3$ & 10.5 & 84.1 & 3.1 & 100 & 1.2 & 100 \\
\hline $2.5,4$ & 8.6 & 94.4 & 4 & 100 & 1.4 & 100 \\
\hline $2.5,5$ & 3.3 & 100 & 2.1 & 100 & 1.4 & 100 \\
\hline$\overline{3,3}$ & 1.7 & 100 & 1.2 & 100 & 0.9 & 100 \\
\hline 3,4 & 3.1 & 100 & 1.7 & 100 & 0.9 & 100 \\
\hline 3,5 & 8.3 & 100 & 2.1 & 100 & 1.2 & 100 \\
\hline
\end{tabular}

\footnotetext{
${ }^{25}$ The bipartisan set is thus the set of options played with a strictly positive probability at the equilibrium.

${ }^{26}$ We let $a$ and $b$ vary by increment of 0.05 respectively in $\left[\frac{-b}{2}, \frac{1-b}{2}\right]$ and $[0,1]$.
} 
Table 2 suggests that the "flatness" and the "skewness" of the distribution of abilities determine whether progressive taxation is likely to emerge in equilibrium. If both $\alpha$ and $\beta$ equal one, the distribution of abilities is uniform (with maximum flatness) and then both regressive and progressive taxes can emerge in equilibrium (although progressivity is more likely). For the beta parameters $\alpha=1$ and $\beta=2$ the distribution becomes linearly decreasing and positively skewed. The shifting of probability mass to the low ability levels makes this group sufficiently large to impose regressive taxes (Recall that in our model the poor prefer marginal regressivity to induce more effort and maximize tax revenues). A simultaneous increase in $\alpha$ and $\beta$ reduces the flatness of the distribution and produces a distribution more tightly concentrated around the middle. This implies an increasingly large middle income group who can thus impose progressivity as a way to reduce its tax burden at the expense of the high-income group. Therefore for sufficiently high $\alpha$ and $\beta$, the set of voting equilibria is confined to progressive taxes. These results suggest that the prevalence of progressive taxation may be due to the predominance of the middle class in the income distribution.

Table 2 also reveals that the uncovered set and the bipartisan set are very discriminating solution concepts. This is in contrast to Epstein (1997) who shows that for games of purely distributive politics the uncovered set coincides approximately with the Pareto set which is typically very large. Our results show that for the model used here (with distortionary taxation), the uncovered set and the bipartisan set can give rather sharp predictions on equilibrium outcomes.

In the next section we introduce repeated elections and study the trajectory of vote-maximizing policies. 


\section{$5 \quad$ Vote-maximizing trajectory}

We use the dynamic version of the two-party electoral competition game due to Kramer (1977). In this voting game, two political parties repeatedly compete for the votes with the peculiarity that when a party is elected, it is committed to keep the same political platform for the next election (reputation inertia). ${ }^{27}$ It is further assumed that parties are interested in maximizing the size of their majority (or net plurality defined as $n\left(t_{1}, t_{2}\right)-n\left(t_{2}, t_{1}\right)$ ), and thus the opposition party will always select a policy which maximizes its voting share given the incumbent's policy. In absence of Condorcet winner, both parties then alternate in office because each party can win the election once it knows the policy chosen by the other party. This dynamic voting process can be represented by the sequence of winning policies, which Kramer calls a vote-maximizing trajectory (i.e., a sequence of policies such that each policy along the sequence beats with a maximal number of votes its predecessor). Formally, for any two adjacent policies $\left(t_{i}, t_{i+1}\right)$ along the sequence, $t_{i+1} \in \operatorname{argmax}_{t \in T} n\left(t, t_{i}\right)$.

Using our model, we have simulated the Kramer trajectories for the various parameters of the beta distribution of abilities. For each pair of parameters, we get that (i) the Kramer trajectory converges to a cycle which is independent of the starting point; and that (ii) a coalition of the rich and the poor alternates with a coalition of the poor and the middle.

The results on the size and composition of the Kramer cycles are reported in Table 2. ${ }^{28}$ According to these simulation results, progressive taxation again emerges as the only possible voting outcome when there is a predominance of the middle class in the distribution of abilities. Indeed the

\footnotetext{
${ }^{27}$ Note that this full commitment assumption stands in sharp contrast with the nocommitment assumption in section 3. Therefore, besides presenting independent interest, this dynamic electoral competition game enables us to test the robustness of our predictions of the voting outcomes to the commitment assumption.

${ }^{28} a$ and $b$ vary by increment of 0.025 respectively in $\left[\frac{-b}{2}, \frac{1-b}{2}\right]$ and $[0,1]$.
} 
beta distribution produces a Kramer cycle that contains only progressive taxes when it is sufficiently concentrated around the middle (with $\alpha$ and $\beta$ high enough). Shifting the probability mass of individual abilities evenly towards the extremes $(\alpha=\beta=1)$ makes the vote maximizing trajectory cycles evenly between regressive and progressive taxes; while a distribution concentrated at low-abilities $(\alpha=1 ; \beta=2)$ induces a Kramer cycle that only contains regressive taxes.

Kramer(1977) has also introduced the minmax set which is defined as the set of policies whose maximal opposition is minimal. Formally, $\min \max (T)=$ $\arg \min _{t} \max _{t^{\prime} \neq t} n\left(t^{\prime}, t\right)$. Kramer has demonstrated that for Euclidean preferences the minmax set behaves like a basin of attraction in the sense that any vote-maximizing trajectory converges to the minmax set. For the model used here (with non-Euclidean preferences), we obtain that Kramer cycles always pass through the minmax set (which may reduce to a singleton).

These results are of course only suggestive since they are based on some simulations of a stylized model. But the fact that similar results emerge from different voting games (see previous sections) leads us to believe that they point out to something more general that would be worth examining in future work.

\section{Conclusion}

This paper is an attempt to determine the conditions under which (marginally) progressive tax schedules emerge as a voting outcome. Given the potential complexity of this problem we had to employ a highly stylized model with quadratic taxation that nevertheless includes the salient aspects of voting over non-linear income tax schedules, namely that taxes affect work incentives and that individuals with different incomes have conflicting interests over tax schedules. Although our modeling of disincentive effect of progres- 
sivity creates a bias in favor of regressive tax schedules, vote cycles over progressive and regressive taxes are almost inevitable in our setting.

In that context we have adopted three different ways to narrow down the set of possible equilibria and to characterize equilibrium outcomes as a function of the underlying distribution of abilities. The first approach reduces the policy space to the tax schedules that are ideal for some voter. In doing so we have obtained a majority winner corresponding to the policy preferred by the median ability individual which in general involves progressive taxes. The second approach eliminates weakly dominated strategies, reducing the set of possible voting equilibria to a relatively small subset. We have shown that this subset contains only progressive taxes when the distribution of abilities is sufficiently concentrated around the middle. The third approach considers a sequential two-party competition game in which parties alternatively propose tax schedules that maximize the size of their majority. We have shown that this dynamic voting game will eventually cycle on a small subset that again contains only progressive taxes when the distribution of abilities is tightly concentrated around the middle.

We do not claim that the particular approaches examined here are the "correct" ones in any sense. The point of this work is that each approach captures a different aspect of reality. By predicting similar voting outcomes it gives some credence to this simulation-based analysis of a highly stylized model..

Acknowledgments. This paper was prepared for presentation at the ISPE conference on Public Finance and Redistribution at CORE, Belgium, June 2000. We wish to thank our discussants Gerard Roland and David Wildasin for helpful comments. We are especially grateful to the two Co-editors, an anonymous referee and to Yan Chen whose suggestions greatly improved the paper. We also thank participants at the Public Economics conference of the AFSE in Marseille, May 2000 and seminar participants at University of California, Irvine and at the Wallis Institute, Rochester. This paper has been initiated while Jean Hindriks was visiting 
the Institut d'Economie Industrielle (IDEI) in Toulouse, and it has been revised while Philippe De Donder was visiting the Wallis Institute of Political Economy at the University of Rochester. Financial support from both institutions is gratefully acknowledged. The usual disclaimer applies. 


\section{References}

[1] Banks, J., J. Duggan and M. Le Breton, 1998, Bounds for mixed strategies equilibria and the spatial theory of elections, mimeo, University of Rochester.

[2] Berliant, M. and M. Gouveia, 1994, On the political economy of income taxation, mimeo, University of Rochester.

[3] Besley, T. and S. Coate, 1997, An Economic Model of Representative Democracy, Quarterly Journal of Economics, 112, 85-114.

[4] Blundell, R., A. Duncan and C. Meghir, 1998, Estimating labor supply responses using tax reforms, Econometrica, 66, 827-861.

[5] Cukierman A., A. Meltzer, 1991, A political theory of progressive income taxation, in Political Economy, by A. Meltzer, A. Cukierman and S.F. Richard.(Eds.). Oxford University Press. New York.

[6] De Donder, P., 2000, Majority Voting Solution Concepts and Redistributive Taxation, Social Choice and Welfare, 17: 601-627

[7] De Donder, P., J. Hindriks, 2002, Majority support for progressive income taxation, mimeo CORE.

[8] De Donder, P., M. Le Breton and M. Truchon, 2000, Choosing from a weighted tournament, Mathematical Social Science, 40: 85-109.

[9] Epstein, D., 1997, Uncovering some subtleties of the uncovered set: social choice theory and distributive politics, Social Choice and Welfare, 15, 81-93.

[10] Gans, J. and M. Smart, 1996, Majority voting with single-crossing preferences, Journal of Public Economics 59, 219-37.

[11] Hettich, W. and S.L. Winer, 1988, Economic and political foundations of tax structure, American Economic Review, 78, 701-13. 
[12] Hindriks, J., 2000, Is there a demand for progressive taxation?, Economics Letters, forthcoming.

[13] Hinich, M., J. Ledyard and P. Ordeshook, 1972, Nonvoting and the existence of equilibrium under majority rule, Journal of Economic Theory, 4, 144-53.

[14] Kramer, G., 1977, A dynamic model of political equilibrium, Journal of Economic Theory, 16, 310-34.

[15] Laffond, G., J.F. Laslier and M. Le Breton, 1993, The bipartisan set of a tournament game, Games and Economic Behavior, 5, 182-201.

[16] Laslier, J.F., 2000, Interpretation of Electoral Mixed Strategies, Social Choice and Welfare, 17-2, 283-292.

[17] Marhuenda, F., I. Ortuno-Ortin, 1995, Popular support for progressive taxation, Economics Letters,48, 319-24.

[18] Miller, N., 1980, A new solution set for tournaments and majority voting, American Journal of Political Science, 24, 68-96.

[19] Myles, G., 2000, On the optimal marginal rate of income taxation, Economics Letters, 113-19.

[20] Osborne, M.J., A. Slivinski, 1996, A Model of Political Competition with Citizen candidates, Quarterly Journal of Economics, 111, 65-96.

[21] Roberts, K., 1977, Voting over income tax schedules, Journal of Public Economics, 8, 329-40.

[22] Roell, A., 1997, Voting over non-linear income tax schedules, mimeo Tilburg University.

[23] Romer, T., 1975, Individual welfare, Majority Voting and the Properties of a Linear Income Tax, Journal of Public Economics, 4, 163-185. 
[24] Roemer, J., 1999, The democratic political economy of progressive income taxation, Econometrica, 67, 1-19.

[25] Snyder, J. and G. Kramer, 1988, Fairness, self-interest, and the politics of the progressive income tax, Journal of Public economics, 36, 197-230.

[26] Young, P., 1990, Progressive taxation and equal sacrifice, American Economic Review, 80, 253-66. 